https://doi.org/10.48009/2_iis_2005_338-344

\title{
SIMPLIFIED PROCEDURES IN DIGITAL VIDEO EDITING: CONCEPTS AND TECHNOLOGICAL ALTERNATIVES
}

\author{
Azad I. Ali, Butler County Community College, azad.ali@bce3.edu \\ Frederick G. Kohun, Robert Morris University, kohun@rmu.edu \\ Gary J. DeLorenzo, California University of Pennsylvania, delorenzo@cup.edu
}

\begin{abstract}
Recent technological changes have significantly simplified digital video editing. Yet, despite this simplification, digital video editing remains intimidating to some and confusing to others. Different reasons were cited for this intimidation, including the confusion on the steps required to edit digital videos and the uncertainty on the concepts and technologies involved. But digital video editing, in its basic form, can be completed by following simple procedures and through the use of hardware and software commonly found on PCs. This paper explains the steps to follow in digital video editing. It also describes some reasons that made digital video editing simple for some but difficult for others. The paper clarifies the steps involved in digital video editing and the available technological alternatives in each step.
\end{abstract}

Keywords: Digital Video Editing, Digital Video, Computer Video Editing, Simple Digital Video Editing

\section{INTRODUCTION}

Until recently, digital video editing was a complicated task and limited to professionals who had the necessary equipment and the required specialty knowledge [5]. Capturing videos, even the simplest ones, and then editing to the desired result required specialized knowledge in hardware and software operations designed specifically for editing videos. Recent technological advances have simplified these steps significantly. Using newer technologies, individuals can follow simple steps to edit digital videos through their basic knowledge of computer operations and application software.

Despite these technological changes, digital video editing remains intimidating to some while mysterious to others. This mystery is attributed to the confusion about the steps involved in digital video editing and the technologies involved in each step [6]. But simple digital video editing does not require substantial knowledge of these technological alternatives. Instead, digital video editing can be completed through the following of simplified step-by-step procedures while having a basic knowledge of the operation required in each step.

This paper clarifies the technological alternatives and procedures involved in simple digital video editing. It explains concepts that are commonly cited in this field and the factors that affect both simple and complex digital video editing. The conclusion section suggests basic steps in digital video editing and the technological alternatives used in each step. 


\section{DIGITAL VIDEO EDITING - SIMPLE CONCEPTS}

This section provides a brief explanation on concepts that are considered basic when associated with digital videos.

Digital Image: A digital image simply means an image or a picture that has been produced with a computer [2]. The machine takes a visual image and translates it into a series of mathematical values or bits of information and stores them in a computer file, hence named digital file. In other words, information about each bit or point on the image is stored in the digital file. If you take the many bits or points that can be found on each picture, it can be understood that the file will contain a lot of information about the image which results in a large digital file size. A similar name used here is electronic image or still image.

Compression: In many cases, certain compression techniques are used to reduce the size of image files. The compression techniques keep the information about the image intact but follow a formula for minimizing the repetition of information about the image so to minimize the size of the file. For example, if a specific color is repeated continuously one thousand times on an image, the same information about the color is saved one thousand times. But with compression, instead of the information repeated many times, a formula that indicates the color, locations of the color and number of times repeated, is used. While compression reduces the file size and provides efficiency during downloading over various bandwidths, it may also lead to loss of picture quality. High compression rates lead to combining similar or close colors or shapes, that is, the higher the compression rate relates to a lower quality of the image.

Digital Video: Digital video is a combination of multiple digital images saved as a frame [6]. The video that we see on the computer is divided into different frames that are combined into one digital file. The digital video is measured in number of frames per second. The standard for frames in digital videos is about 20 to 30 frames per second [5]. The higher the number of frames per second makes the quality of the video better. A high number of frames per second in a video decrease the gaps between one frame and the next, thus, increasing the quality of the view produced. Editing of digital video is made easier because of the multiplicity of frames in typical digital video files. During the process of editing the video, the editor can break down the video into individual frames and then insert, delete, and update individual frames as required to meet the desired result.

Analog Video: While a digital video is divided into numerous frames, an analog video displays images similar to digital, but the storage format is different [6]. The video file in analog videos are not divided into frames such as in digital video files. The video file is stored in one unit with no gaps (i.e., no frames) between them. Thus, when editing the analog video, the editor cannot take the video and break it easily into individual pieces (frames). Instead, different methods are employed to edit one continuous unit of video images. Based on the apparent differences between the two formats of video files, it can be said in general terms that digital videos result in better quality display while at the same time providing easier video editing capabilities. 


\section{DIGITAL VIDEO EDITING - SIMPLICITY REASONS}

Recently, digital videos have become more widely used than their analog counterpart. This increase is attributed to the growth in digitized-based computer technology throughout the world and the different factors that made editing digital video easier such as newer technological advancements. This section explains specific changes in technologies that simplified the task of capturing, editing and deploying digital videos.

\section{Improvement in Storage and Processing Devices}

Editing digital videos usually requires copying digital files to a hard drive, editing the file, and copying it back to another media [1]. A typical half-hour video may require one gig of hard drive space depending on the compression and the many additional features within the video. These files require substantial storage space at three fronts: when capturing the video on the digital camera, when transferring them to the computer, and when copying them to another media. Also, these files require substantial speed in processing large files from one medium to another; otherwise, video editing would become a very slow process and discourage users throughout the process.

Recent technological improvements have overcome space and processing constraints. Storage devices and capacities are abundant, processor speeds for all devices have improved dramatically and prices for the various media have dropped [5]. This means significantly cutting the time it takes to capture the video, transforming the captured video, and redeploying the video back to another media.

\section{Improvement in Video Editing Software}

Video editing software packages have become more abundant, less expensive, and easier to use. For example, Microsoft Windows XP comes installed with Windows Movie Maker [4]. Other software solutions such as Adobe Premiere and Pinnacle Systems are examples of video editing software that are sold for about $\$ 100$. These packages have significant online help, tutorials, and other screen tips and support features that make the process of learning about digital video editing easier [5].

\section{Enhancement in Transferring Technology}

To meet the challenge of copying digital video files from a digital camera to the computer and back to other devices requires computers that are equipped with ports to connect to external devices through communication media. The ports and the communication media must both be able to handle the transfer of large video files at a high processing speed.

Recent advancement in technologies has helped with this requirement. Newer PCs come preequipped with different ports like USB, FireWire, and serial ports [1] as standard configuration devices. Computer communication through transmission media is also made easier. In most cases, it requires nothing more that a simple cable connection from the computer that detects the 
hardware attached to it, such as a digital camera, and automatically actives the application software programs to operate it.

\section{DIGITAL VIDEO EDITING - DIFFICULTY REASONS}

Despite the recent enhancement of digital video technology, there are factors that sometimes make digital video editing difficult, thereby deterring someone from working on or editing digital videos. This section explains some of these factors.

\section{Complicated Terms}

Despite the attempt to clarify and simplify technological terms, there are many "coded terms" to understand with when researching, buying, and using digital video editing solutions. First, digital cameras have ambiguous names that refer to the technology with acronyms and numbers. For example: DZ-MV1, M2, GR-DVL9500U, and DCR-TRV510 are brand names of digital cameras. Books, magazines and products use terms with digital video editing such as lines of resolution, color signal, and signal-to-noise ratio to have little context and meaning to novice editors. These terms do not give a good definition and understanding about the meaning of the technology and may give the impression that the process of editing digital video is difficult and complicated [6].

\section{Copying and Processing Time}

The time it takes to complete a task in digital video editing is crucial to making it appealing to editors. If it takes too long to complete a task, then it may discourage editors from pursuing the task. As noted earlier, digital video files are large; thus, it takes time to transfer the files from a digital camera to the hard drive, edit the file, and copy the final edited file back to the digital camera or other devices. Despite improvements in file transfer protocol technology, copying and editing digital video files remains a slow process.

\section{Complicated Software Tools}

The software editing tools that are available for video editing frequently comes equipped with many features that are designed for people knowledgeable in video editing [7]. These features are confusing to novices or for those editors working on simple editing techniques. For example, the long list of images displayed at the bottom of the screen and the different timelines may confuse novice editors. Besides, the digital video file size complicates processing efficiency further such as errors in manipulating the frames or the slow editing and processing time may cause the dreaded hour glass to show for an extended period of time.

\section{DIGITAL VIDEO EDITING - SIMPLIFIED STEPS}

In most cases, digital video editing is not a single step. Instead, digital video editing involves capturing the video, transferring the video to the computer, editing it using software editing tools and then redeploying it to other devices. This section explains the steps within the overall process. 


\section{Capturing the Video}

A scene such as a wedding or a birthday party can be captured using a video camera where the actual content of the event is saved on a storage device. Three different storage devices are commonly used with a video camera: Mini DV, 8mm tape and VHS. Of these devices, the Mini DV is the only one that records videos in digital formats. A mini DV can store up to 11 gig of digital video [6]. The other two devices store the video in analog format, but as noted earlier, the analog format becomes cumbersome for future editing. Recently, some newer cameras allow the use of a CD or a DVD for video capturing.

While editing features are included within some digital camera settings, these editing features are limited. In most cases, the video is transferred into the computer's hard drive for ease of use for better editing.

\section{Transferring to the Computer}

Digital video cameras hold the video in a digital file. To take advantage of a computer's processing and editing features, the digital file needs transferred to a computer. One of three transmission media is commonly used for the transfer: FireWire, USB and RCA connectors [6].

FireWire. The FireWire or IEEE1394 cable is the fastest of the three that transfers digital videos from digital cameras to computers in digital formats [1]. The FireWire is plugged into both the computer and the digital camera. Software on the computer detects the digital camera connectivity and activates a program that offers an option to transfer the digital video to the computer.

USB. The USB cable is a popular option for transferring digital videos to the computer. Although slower than the FireWire option, it offers a fast media for transferring digital videos and maintaining the digital format for the file [1]. Newer PCs come with multiple USB ports where some of these ports are available at the front panel of the computer.

RCA Connectors. The RCA connectors come with red, yellow and white wires used to transfer the digital file as video and audio signals. A special card is attached to a USB port or COM serial port on the computer where the RCA connectors are attached to the digital camera and the card. The RCA connection is slower in processing and it transforms the digital camera file into analog format [6].

\section{Digital Video Editing}

After the digital video file is saved on the computer, the file can be edited using software tools. Although different software packages are available for this purpose, they have common features that make simple editing of digital videos closely similar [3].

Video editing software packages usually display the digital file in two views: storyboard and timeline. As noted earlier, a digital video contains many frames that are individual pictures displayed in succession to give the impression of moving video. So a digital video that is one 
hour long (3600 seconds/hour) could contain as many as 216,000 frames. In the storyboard view of the video, individual frames (pictures) are placed on the storyboard that can be manipulated. Individual picture frames can be cut, pasted or moved around as needed by the user. Special effects can also be applied to selected frames [5]. The timeline view edits the video according to a time frame. So users can add titles between time slices (for example between 5 and 6 minutes) of the video or add a transition or special sound as the video reaches a specific time.

Common editing features that are available with both timeline and storyboard views include the following:

Cut and paste. Using the software, users can cut different pieces of the video. The timeline toolbar is used for cutting pieces of the video in time segments. For example, users can cut between 5 minutes and 10 minutes of the video. Users can also cut video frames in the storyboard view. In this view, individual frames of the video are shown where users can cut, copy, or move frames to different places in the video file.

Transition editing. These are the editing features that can be applied when transitioning from one frame to another or from one segment of the video to another. The common transitional effects that are usually applied when editing videos include checkerboard effect, circles across, bars, box out whether diagonal or vertical, dissolve, and fade in/out [6].

Apply titles. Titles can be added to individual frames, to a group of frames, or to a particular time segment of the video [6]. Titles can be added to the video at the beginning of the movie, before selected clips on the storyboard, on the selected clips on the storyboard, and as credits at the end of the movie.

\section{Redeploying the Video}

Digital video editing software packages come with features that allow for saving the digital video to other media such as a CD or a DVD. If the software does not offer this feature of deploying the video, a digital video file can be copied to a CD or DVD using CD burning software such as Roxio's Easy Media Creator.

Copying. Some video editing software includes features that allow for transferring the final, edited video back to a digital camera or transferring the edited video onto a VHS tape. For the VHS tape transfer, the analog transfer media using RCA connectors are used to transfer the video.

DVD authoring. Users who have a need to copy digital videos to a DVD must have a computer equipped with a DVD writer. DVD offers a high storage capacity (up to 9 gig) where users can store more than 4 hours video on the DVD. The quality of the DVD video is also higher than other media.

Burning CD. Newer computers with CD read/write drives normally have CD burner software installed on them. The storage capacity of the CD is about $650 \mathrm{Meg}$ and users can record up to 
about 40 minutes of video on a typical CD. Second, the quality of the video recorded on a CD is less than those recorded on a DVD.

\section{SUMMARY}

As noted in this paper, digital video editing has become easier due to advancements in hardware and software technology of computers while streamlining the processing steps for users. While improvements have been made, digital video editing sometimes remains confusing and intimidating to users. These difficulties can be related to the many technological terms that are used in digital video editing and a lack of understanding on the steps to take to capture, transfer, and edit digital video files. But once the processing steps are simplified and the technical terms are understood by users, then the goal to edit digital videos can be realized by different computer users who have the basic knowledge of the technology.

\section{REFERENCES}

1. Evans, A., Kendall, M. \& Poastry, M. A. (2005). Technology in Action: Complete. Upper Saddle River, NJ: Prentice Hall.

2. Krejcarek, P. (2003). An Introduction to Digital Imaging With Photoshop 7. Clifton Park, NY: Thompson Delmar Learning.

3. Mainelli, T. \& Stafford, A.( Jan 2005). Inexpensive Video Editors Shine: These $\$ 100$ apps offer enough powerful features to make your next home move a masterpiece. PC World, 23:1, 52-53.

4. Microsoft. Create Digital Home Movies With Windows Movie Maker in Windows XP. Retrieved March $2^{\text {nd }}, 2005$ from http://www.microsoft.com/windowsxp/using/moviemaker/default.mspx.

5. Ozer, J. (Jan 2005). "Making Movies: Learn How to Edit Video and Create ProfessionalLooking DVDs". PC Magazine, 24:1,.147-161.

6. ShortCourses. A Short Course in Digital Video. Retrieved March $2^{\text {nd }}, 2005$ from http://www.shortcourses.com/video/

7. SimplyDV.Com. The no-nonsense Guide to Choosing and Using Digital Video. Retrieved from http://www.simplydv.co.uk. 